\title{
Measurements of CO concentration distribution for Mars atmospheric entry by combining OES and TDLAS
}

\author{
Lin Xin $^{\text {ab }}$, Chen Lianzhong ${ }^{\mathrm{a}}$, Ou Dongbin ${ }^{* \mathrm{a}}$, Li Fei $^{\mathrm{b}}$, Yu Xilong ${ }^{\mathrm{b}}$ \\ ${ }^{a}$ Beijing Key Laboratory of Arc Plasma Application Equipment, China Academy of Aerospace \\ Aerodynamics, Beijing 100074, China; ${ }^{b}$ State Key Laboratory of High Temperature Gas Dynamics, \\ Institute of Mechanics, Chinese Academy of Sciences, Beijing 100190, China
}

\begin{abstract}
Shock tube experiments are carried out to study the physical and chemical processes during a vehicle entry into the Mars atmosphere using optical emission spectroscopy (OES) and tunable diode laser absorption spectroscopy (TDLAS). Gas temperature and $\mathrm{CO}$ concentration distribution are diagnosed behind a shock wave in a $\mathrm{CO}_{2}-\mathrm{N}_{2}$ mixture with two different conditions of initial pressure and velocity. The strong shock wave is established in a shock tube driven by combustion of hydrogen and oxygen. Time-resolved spectra of the $\Delta v=0$ sequence of the $B^{2} \Sigma^{+} \rightarrow X^{2} \Sigma^{+}$electronic transition of $\mathrm{CN}$ have been observed through OES. A precise analysis of the $\mathrm{CN}$ violet spectra is performed and used to determine rotational and vibrational temperatures. Two absorption lines in the first overtone band of CO near $2.33 \mu \mathrm{m}$, are selected from a HITRAN simulation to calibrate laser wavelength and detect the $\mathrm{CO}$ concentration. Combined with these temperature results using OES, CO concentrations in the thermal equilibrium region are derived, which are $2.91 \times$ $10^{12} \mathrm{~cm}^{-3}$ and $1.01 \times 10^{13} \mathrm{~cm}^{-3}$, corresponding to equilibrium temperatures equal to $7000 \pm 400 \mathrm{~K}$ and $6000 \pm 300 \mathrm{~K}$ in low and high pressure conditions, respectively.
\end{abstract}

Keywords: optical emission spectroscopy (OES), tunable diode laser absorption spectroscopy (TDLAS), shock wave, temperature, concentration

\section{INTRODUCTION}

Mars is one of the most attractive and ambitious objectives for future manned space exploration ${ }^{1-3}$. When a spacecraft enters the Mars atmosphere, a strong bow shock will generate in front of the heat shield of the vehicle. In the shock layer, physical and chemical phenomena produce a non-equilibrium situation giving birth to a strong radiation from the excited species $^{4-7}$. The chemical composition of the Martian atmosphere has been determined to be $95.3 \% \mathrm{CO}_{2}, 2.7 \% \mathrm{~N}_{2}$ and $1.6 \%$ Ar. It is well known that in those conditions the strong radiators are mainly consist of $\mathrm{C}_{2} \mathrm{C}_{2}, \mathrm{CO}$, and $\mathrm{CN}$. The radiation flux generated by these species will make a significant contribution to the entry heating environment for future crewed missions towards Mars, which will be larger and likely enter at higher velocities. Therefore, an accurate understanding of the physical and chemical processes in the shock layer is extremely important in a number of applications, e.g., design and validation of thermal protection systems.

Different groups from USA, Europe, Australia, Japan, and Russia performed a number of experiments on the physical and chemical processes behind a reentry shock wave in Martian-like mixtures, especially about non-equilibrium radiation and chemical reactions ${ }^{8-13}$. Up to now studies, the radiation measurements have been widely studied through optical emission spectroscopy (OES), and several chemical kinetic models have been established. All these results are very helpful to validate relevant chemical models and analysis Mars atmosphere entry processes. However, many questions remain on the physical and chemical models, especially on the mechanisms of thermochemical relaxation and chemical reaction ${ }^{14-16}$. During high-speed entry into Mars atmosphere, the dissociation of $\mathrm{CO}_{2}$ is one of the most important reactions because it is the first chemical reaction to occur behind a shock wave and it affects the whole chemical reactions ${ }^{17,18}$. Temperature is another crucial thermodynamic quantity in shock-induced chemistry, especially the rotational temperature, which controls the collision probability of the gaseous particles in a chemically reacting hot gas $^{19-21}$. Therefore, accurate quantitative measurement of the number density of carbon monoxide and temperature after the strong shock wave will help to study carbon dioxide dissociation and validation the relevant chemical kinetic models.

* Corresponding author: oudongbin@sina.com

Third International Symposium on Laser Interaction with Matter (LIMIS 2014), edited by

Yury M. Andreev, Zungi Lin, Xiaowu Ni, Xisheng Ye, Proc. of SPIE Vol. 9543, 954314

(c) 2015 SPIE · CCC code: 0277-786X/15/\$18 - doi: 10.1117/12.2180592 
Experiments in this field are usually performed with shock tubes, the conditions in which are the closest possible to the real conditions of entry into the planetary atmosphere. In our studies, a hydrogen-oxygen combustion driven shock tube was used with two initial pressure and velocity conditions. $\mathrm{CO}$ concentration and gas temperature distribution behind a strong shock wave were simultaneously measured by an optical diagnostic system based on OES and TDLAS. Temperature and chemical relaxation data were obtained through a precise analysis of high temporal and spatial resolution experimental spectra of $\mathrm{CN}$ violet system $\left(B^{2} \Sigma^{+} \rightarrow X^{2} \Sigma^{+}, \Delta v=0\right.$ sequence). The TDLAS system was operated in scanned-wavelength direct absorption mode to diagnose one $\mathrm{CO}$ absorption line near $2335.778 \mathrm{~nm}$, which was simultaneously calibrated by the known position of the strong CO absorption line near $2335.544 \mathrm{~nm}$ at a room temperature reference cavity. Combined with temperature results using OES, CO concentration distributions in the thermal equilibrium region were determined for the concerned test state. The current results will be very useful to understand $\mathrm{CO}_{2}$ dissociation and provide reliable verification data for assessment of relevant thermochemical models.

\section{THEORY OF ABSORPTION SPECTROSCOPY}

The fundamental theoretical principle of direct-absorption spectroscopy is well understood ${ }^{22}$, and only a brief description is presented here to define our notation. When a well collimated laser at frequency $v\left[\mathrm{~cm}^{-1}\right]$ enters a gas sample with a total path length of $L[\mathrm{~cm}]$, a proportion of light is absorbed and the fractional transmission is described by the Beer-Lambert law expressed as

$$
\left(\frac{I_{t}}{I_{0}}\right)_{v}=\exp \left(-P_{C O} \cdot S(T) \cdot \phi(v) \cdot L\right)
$$

where $I_{0}$ and $I_{t}$ are the transmitted and incident laser intensities, $P_{C O}$ [atm] is the partial pressure of absorbing species, $S(T)\left[\mathrm{cm}^{-2} \mathrm{~atm}^{-1}\right]$ and $\phi(v)[\mathrm{cm}]$ are the line strength and line-shape function, respectively. The line strength $S(\mathrm{~T})$ is a function of temperature as follows

$$
S(T)=S\left(T_{0}\right) \frac{Q\left(T_{0}\right)}{Q(T)}\left(\frac{T_{0}}{T}\right) \exp \left[-\left(\frac{h c}{k}\right)\left(E_{1}^{\prime \prime}-E_{2}^{\prime \prime}\right)\left(\frac{1}{T}-\frac{1}{T_{0}}\right)\right] \frac{\left[1-\exp \left(-\frac{h c v_{0}}{k T}\right)\right]}{\left[1-\exp \left(-\frac{h c v_{0}}{k T_{0}}\right)\right]}
$$

where $h[\mathrm{~J} \cdot \mathrm{s}]$ is Planck's constant, $k\left[\mathrm{~J} \cdot \mathrm{K}^{-1}\right]$ is Boltzmann's constant, $c\left[\mathrm{~cm} \cdot \mathrm{s}^{-1}\right]$ is the speed of light, $E^{\prime \prime}\left[\mathrm{cm}^{-1}\right]$ is the lower-state energy, $v_{0}\left[\mathrm{~cm}^{-1}\right]$ is the line-center frequency, $T_{0}[\mathrm{~K}]$ is the reference temperature (usually $\left.296 \mathrm{~K}\right), Q(T)$ is the partition function of the absorbing molecule $\mathrm{CO}$. The partition function determines the equilibrium population fraction in the lower state as a function of temperature ${ }^{23}$

$$
Q(T)=\sum_{e l} \sum_{v^{\prime \prime} J^{\prime \prime}} g_{e l}\left(2 J^{\prime \prime}+1\right) \exp \left[-\frac{h c E\left(e l, v^{\prime \prime}, J^{\prime \prime}\right)}{k T}\right]
$$

Where " $e l$ " indicates summation over the electronic states, $v$ " is the lower state vibrational quantum number, $J$ " is the lower state rotational quantum number, and $g_{e l}$ is the degeneracy number of the electronic states.

The absorbance $\alpha_{v}$ is defined as

$$
\alpha_{v} \equiv-\ln \left(\frac{I_{t}}{I_{0}}\right)_{v}=P_{C O} \cdot S(T) \cdot \phi(v) \cdot L
$$

The line-shape function $\phi(v)$ is usually approximated using a Voigt profile characterized by the Gaussian and Lorentz profiles. Since the line-shape function $\phi(v)$ is normalized to unity (defined as $\int \phi(v) d v \equiv 1$ ), the integrated absorbance $A\left[\mathrm{~cm}^{-1}\right]$ can be expressed as

$$
A=\int \alpha_{v} d v=P_{C O} \cdot S(T) \cdot L
$$


Therefore, if the temperature is acquired and the wavelength scans strategy is performed, by integrating absorbance over the whole wavelength range, the absorbing pressure of carbon monoxide can be calculated

$$
P_{C O}=\frac{A}{S(T) \cdot L}
$$

Based on the ideal gas equation, the $\mathrm{CO}$ concentration $n_{c o}$ can be deduced

$$
n_{C O}=\frac{6.02 \times 10^{23} \cdot P_{C O}}{R \cdot T} m^{-3}=\frac{6.02 \times 10^{17} \cdot A}{R \cdot T \cdot S(T) \cdot L} \mathrm{~cm}^{-3}
$$

\section{FACILITY AND EXPERIMENTAL SET-UP}

The experimental investigations are conducted in simulated Mars atmospheres behind strong shock waves in a hydrogen-oxygen combustion shock tube facility. Figure 1 gives an overview of the facility with useful dimensional details and the optical diagnostic system. The shock tube is an $8.5 \mathrm{~m}$ long facility consisting of two sections (driver and driven), initially separated by an aluminum diaphragm ( $2 \mathrm{~mm}$ thick). The driver section has an inner diameter of $84 \mathrm{~mm}$, and that of the driven shock tube is $78 \mathrm{~mm}$. Using the combustion product gases at high pressure as the driver gases, a strong shock wave that passes through the stagnant test gas can be easily acquired. The shock wave velocity is measured by three ion probes which are located on the shock tube. The variation of the velocity in these experiments does not exceed $1.3 \%$. Experiments have been carried out in the mixture constituted of $70 \% \mathrm{CO}_{2}$ and $30 \% \mathrm{~N}_{2}$, despite the difference from the exact composition of the Mars atmosphere, such a ratio allows the $\mathrm{CN}$ radicals emission signal-to-noise (SNR) to be significantly improved whereas the chemical kinetics processes remain the same. Based on the previous experiments ${ }^{24}$, two initial pressure/shock velocity conditions are investigated: $P_{i}=100 \mathrm{~Pa}$ and $V_{s}=7.09 \pm$ $0.05 \mathrm{~km} / \mathrm{s}$ (called the low-pressure condition), and $P_{i}=300 \mathrm{~Pa}$ and $V_{s}=5.68 \pm 0.07 \mathrm{~km} / \mathrm{s}$ (called the high-pressure condition).

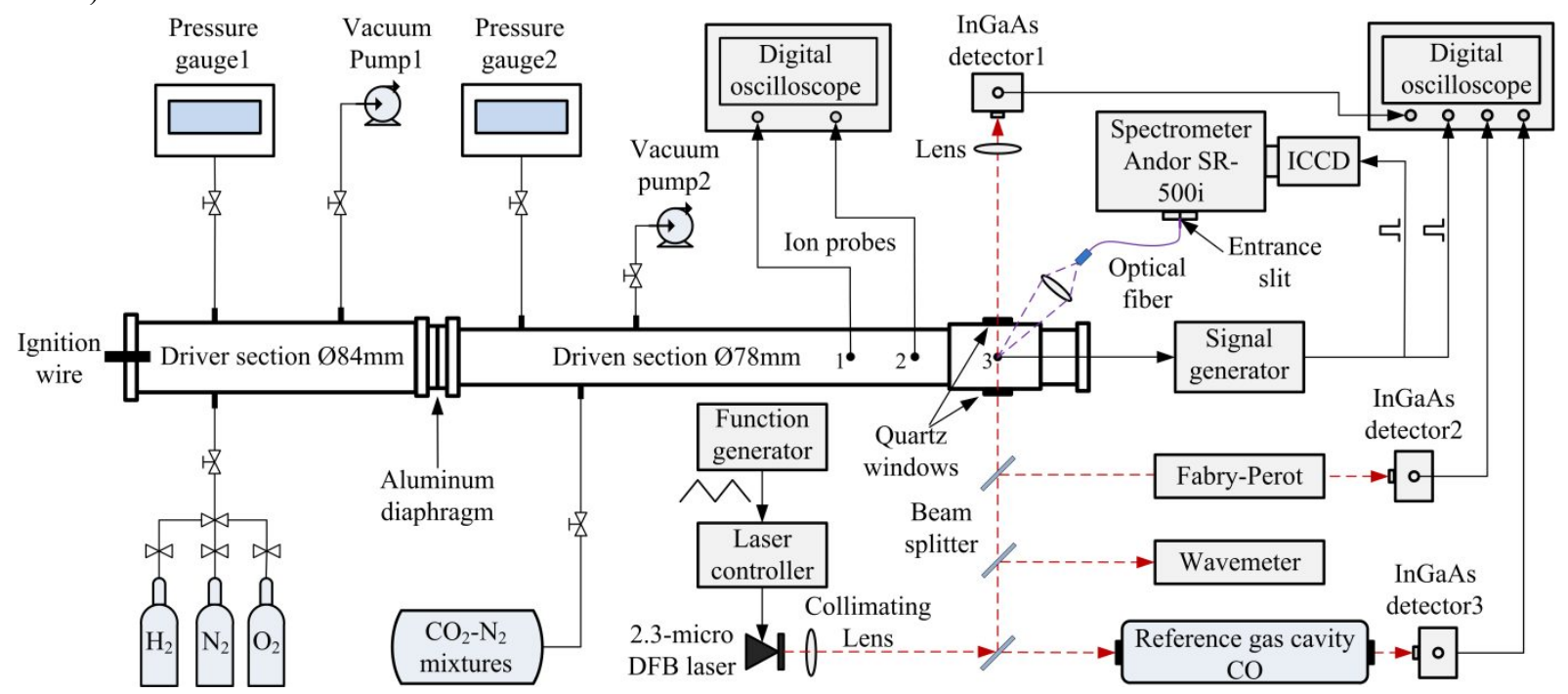

Fig. 1 Schematic diagram of the experimental set-up of the shock tube and the optical experiments arrangement

The optical diagnostic system consists of two parts: the OES system and the TDLAS system. The OES system is designed to allow the recording of the $\mathrm{CN}$ violet system radiation emitted from the high-temperature test gas behind the strong shock wave. The emission has been collected through an optical fiber which is focused the light coming through a lens system. Emission spectra are recorded using an Andor SR-500i spectrograph of focal length $500 \mathrm{~mm}$. Although this spectrometer provides the capability of switching between three different gratings, our experiments utilize uniquely the $1200 \mathrm{~g} / \mathrm{mm}$ grating blazed at $300 \mathrm{~nm}$. The spectrometer is equipped with an Andor DH-740 2048 $\times 512$ pixels gated intensified CCD (ICCD) camera, which can capture spectra with high time resolution (30 ns for our experiments). The entrance slit is fixed at $100 \mu \mathrm{m}$ to get an acceptable SNR and a sufficient spectral resolution. By using a narrow line width diode laser (center wavelength: $777.19 \mathrm{~nm}$, line width $\Delta \lambda<0.001 \mathrm{~nm}$ ), the spectral resolution is measured and is 
equal to $0.154 \mathrm{~nm}$ with a Lorentz shape. The acquired spectra are calibrated in relative intensity in the UV range (below $400 \mathrm{~nm}$ ) by means of a NIST traceable quartz-tungsten-halogen standard reference lamp (Oriel, Model: 63945).

For the TDLAS system, a distributed-feedback laser (manufactured by Nanoplus GmbH), which is controlled by a commercial temperature and current controller (Thorlabs, Model: ITC-502), is lasing the wavelength near $2.33 \mu \mathrm{m}$. A scanned-wavelength direct absorption is used in present study. The injection current of the laser controller is modulated by a triangle ramp signal from a function generator (Tektronix, Model: AFG-3101) so that the lasing wavelength scans a spectral range of $0.7 \mathrm{~nm}$ to probe the $\mathrm{CO}$ absorption transitions as shown in Tabel 1 . The wavelength of the diode laser is modulated at a repetition rate of $50 \mathrm{kHz}$. In order to calibrate and monitor the wavelength of the diode laser simultaneously, the laser beam is separated by three beam splitters. On the one hand, the laser beam travels through a room temperature reference cavity and a free-space mid-IR wavelength meter (Bristol, Model: 721) for absolute wavelength calibration, and on the other hand, the beam travels through a Fabry-Perot (Thorlabs, Model: SA200-18B) interferometer having $1.5 \mathrm{GHz}$ free spectral range (FSR) for relative wavelength calibration.

Table 1. Spectroscopic Line Parameters Used in the Current Study

\begin{tabular}{|l|l|l|l|l|}
\hline Transitions & $\begin{array}{c}\text { Wavelength } \lambda \\
(\mathbf{n m})\end{array}$ & $\begin{array}{c}\text { Frequency } \\
\boldsymbol{v}_{\mathbf{0}}\left(\mathbf{c m}^{-\mathbf{1}}\right)\end{array}$ & $\begin{array}{c}\text { Line Strength } \boldsymbol{S} \mathbf{\mathbf { a }} \\
\mathbf{2 9 6 K}\left(\mathbf{a t m}^{-1} \mathbf{c m}^{-\mathbf{2}}\right)\end{array}$ & $\begin{array}{c}\text { Low-State Energy } \boldsymbol{E}^{\prime \prime} \\
\left(\mathbf{c m}^{\mathbf{1}} \mathbf{)}\right.\end{array}$ \\
\hline 1 & 2335.544 & 4281.657 & $7.916 \times 10^{-2}$ & 57.670 \\
\hline 2 & 2335.778 & 4281.228 & $1.483 \times 10^{-7}$ & 3378.954 \\
\hline
\end{tabular}

The OES system and TDLAS system are triggered simultaneously by an output signal of No. 3 ion probe through a signal generator (Stanford Research System, Model: DG535) when the shock wave nears the location. The camera exposure time is set to $30 \mathrm{~ns}$, which is short enough to "freeze" the shock image within the spectral capture field. Finally, two high dynamic oscilloscopes (Tektronix Inc., Model: DPO4034) are employed for recording the signals from ion probes, InGaAs detectors and the signal generator at a sample rate of $100 \mathrm{MHz}$.

\section{RESULTS AND DISCUSSIONS}

\subsection{Rotational and vibrational temperatures determination}

The $\mathrm{CN}$ radical has been identified as a strongly radiating molecular and one of the main sources of radiation during entries to Mars, which has $\mathrm{CO}_{2} / \mathrm{N}_{2}$ atmosphere. Therefore, it is feasible and practical in estimating the temperature using $\mathrm{CN}$ radicals at relevant conditions for Mars atmospheric entry. In our previous studies, the spectral structure of $\mathrm{CN}$ violet system $\left(B^{2} \Sigma^{+} \rightarrow X^{2} \Sigma^{+}, \Delta v=0\right.$ sequence) has been deeply investigated and a theoretical synthetic spectrum has been calculated based on our spectral resolution. The value of the spectral resolution is $0.154 \mathrm{~nm}$ with Lorentz profile. Even though such a resolution does not allow observing the fine rotational structure of the spectra, the rotational temperature still can be determined by comparing the range $(387.6 \mathrm{~nm}-388.6 \mathrm{~nm})$ of the $\mathrm{CN}$ violet system between the experimental and theoretical synthetic spectra ${ }^{19}$. Conversely, the vibrational structure of $\mathrm{CN}$ violet band system $\left(B^{2} \Sigma^{+} \rightarrow X^{2} \Sigma^{+}, \Delta v=0\right.$ sequence) is apparent and the vibrational temperature is estimated by minimizing the difference between the experimental and theoretical spectrum. A dimensionless quantity $E r$ is used to describe the measurement accuracy. More details and uncertainty analysis description of this method can be found in the literature ${ }^{21}$.

Temporal behavior of the $\mathrm{CN}$ violet system $\left(B^{2} \Sigma^{+} \rightarrow X^{2} \Sigma^{+}, \Delta v=0\right.$ sequence) behind strong shock waves are observed through OES. Figure 2 and 3 present some comparisons of experimental spectra with calculated spectra when non-equilibrium and equilibrium are assumed to be achieved, respectively. In Fig. 2 and 3, the rotational and vibrational temperatures of the $\mathrm{CN}$ radical are obtained by fitting the profile of the experimental spectra of the $\mathrm{CN}$ violet system $\left(B^{2} \Sigma^{+} \rightarrow X^{2} \Sigma^{+}, \Delta v=0\right.$ sequence) to that of the theoretical spectra. The profiles in the solid line represent the experimental spectra of the $\mathrm{CN}$ violet system at different delay times. The profiles in the dotted line are the corresponding theoretical synthetic spectra with the best-fit rotational and vibrational temperatures. 

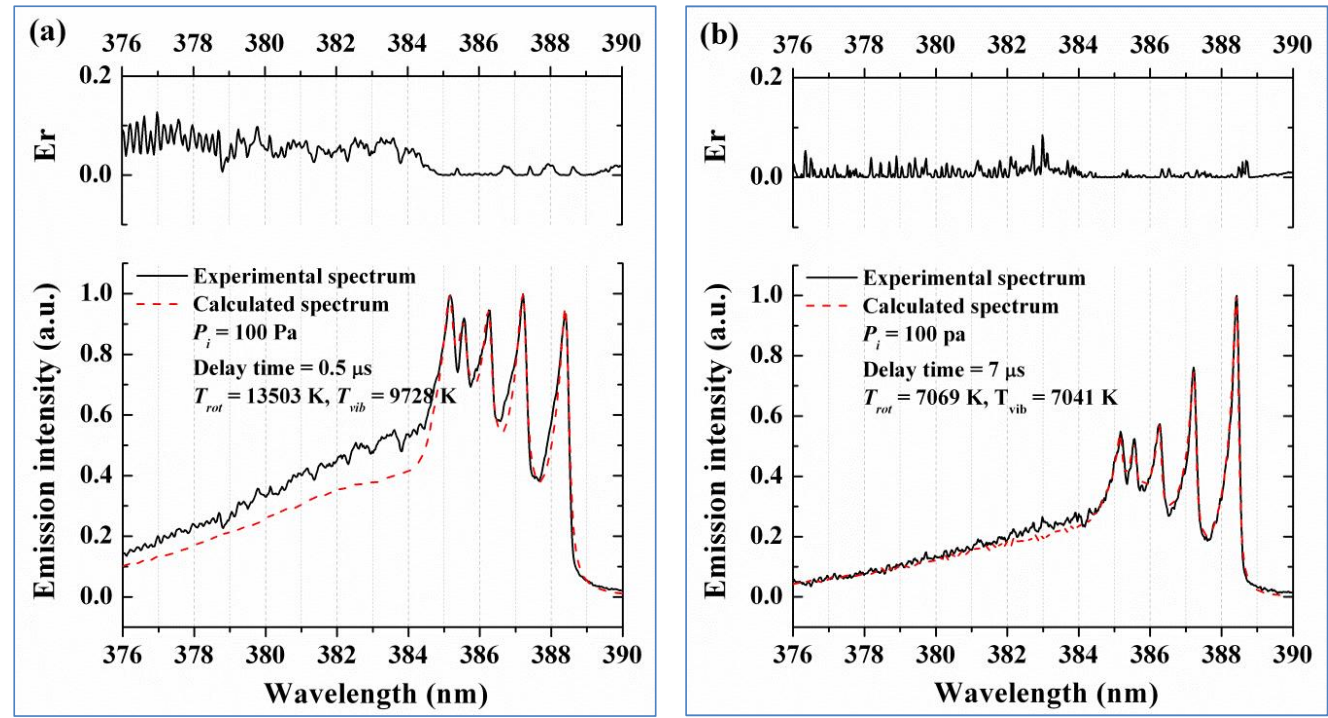

Fig. 2 Experimental and theoretical synthetic spectra of $\mathrm{CN}$ violet system $(\Delta v=0)$ at different delay times (Experiment: $P_{i}=$ $100 \mathrm{~Pa}, V_{s}=7.09 \pm 0.05 \mathrm{~km} / \mathrm{s}$. Composition: $70 \% \mathrm{CO}_{2}$ and $30 \% \mathrm{~N}_{2}$ )
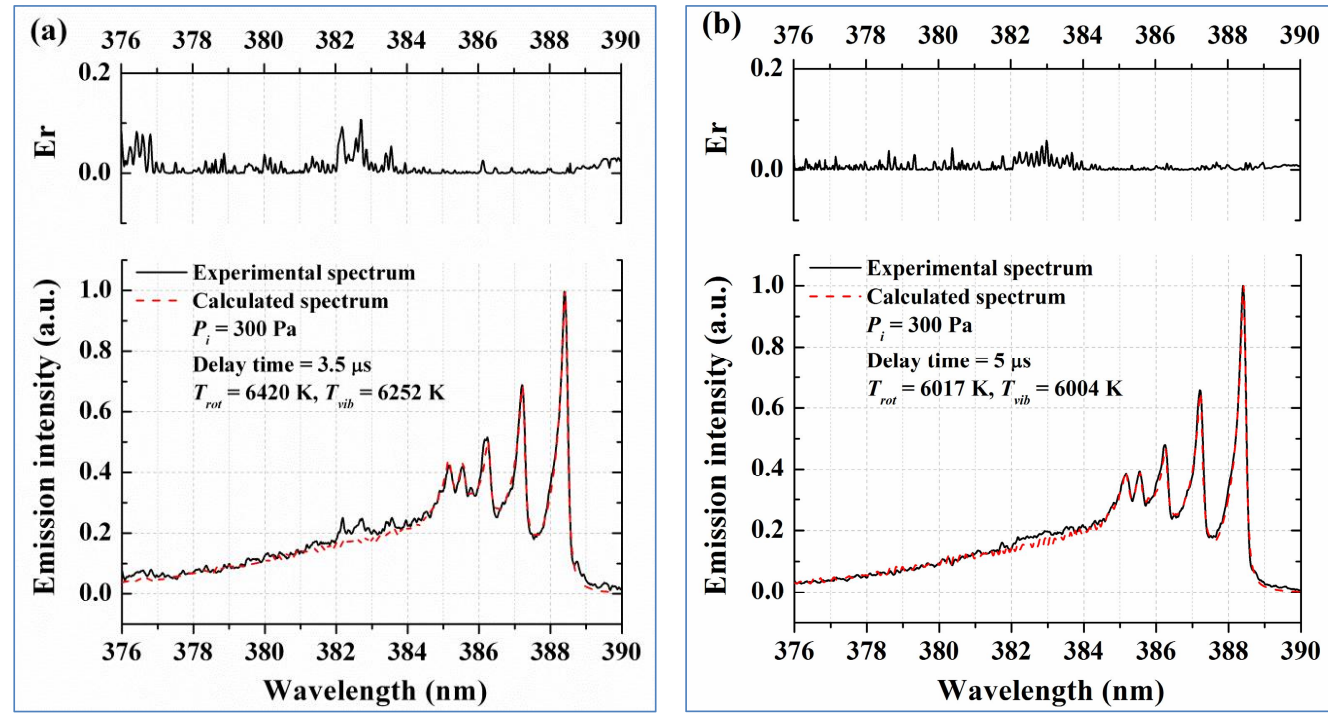

Fig. 3 Experimental and theoretical synthetic spectra of $\mathrm{CN}$ violet system $(\Delta v=0)$ at different delay times (Experiment: $P_{i}=$ $300 \mathrm{~Pa}, V_{s}=5.68 \pm 0.07 \mathrm{~km} / \mathrm{s}$. Composition: $70 \% \mathrm{CO}_{2}$ and $30 \% \mathrm{~N}_{2}$ )

Figure 2a shows excellent agreement between the theoretical synthetic and experimental spectra in the wavelength range of $385 \mathrm{~nm}-390 \mathrm{~nm}$ and that means reasonable rotational temperature is achieved. But for wavelength in the range of $376 \mathrm{~nm}-385 \mathrm{~nm}$ (the tail region of $\mathrm{CN}$ violet system), the theoretical spectra do not agree well with experimental spectra, which indicates that the vibrational state of $\mathrm{CN}$ violet system is not to have a Boltzmann distribution for high $J$ rotational lines. However, the vibrational temperature determination can still keep satisfactory precision. This is because the tail region of spectra is unlikely to have large influence on vibrational temperature determination due to much lower energy quotient in high $J$ rotational line states compared with energy quotient in low $J$ rotational line states. In the quasi equilibrium and equilibrium region, as shown in Fig. 2b and Fig. 3a, b, the $E r$ indicates that the theoretical synthetic and experimentally measured spectra match up very well and that reasonable rotational and vibrational temperatures have been obtained. 


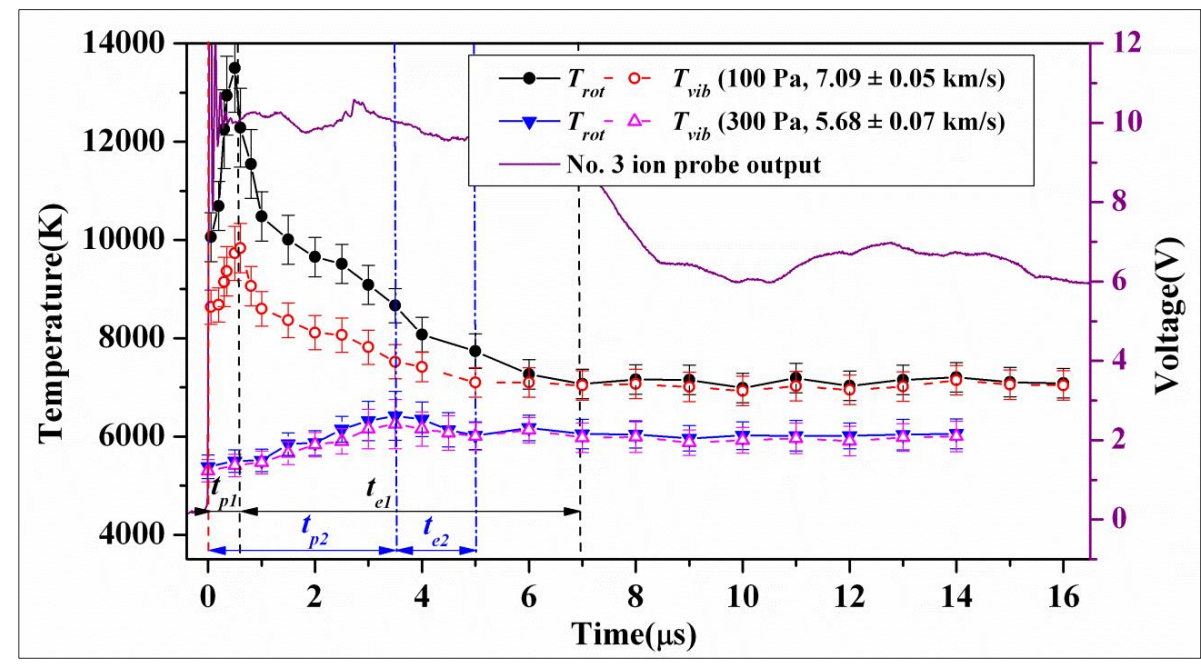

Fig. 4 Time evolutions of rotational and vibrational temperatures behind the shock waves for the two initial pressure conditions (Composition: $70 \% \mathrm{CO}_{2}$ and $30 \% \mathrm{~N}_{2}$ )

Temporal profile of the temperatures behind the shock wave is inferred through the precise analysis of time gating spectral of $\mathrm{CN}$ violet system $\left(B^{2} \Sigma^{+} \rightarrow X^{2} \Sigma^{+}, \Delta v=0\right.$ sequence). Figure 4 presents the evolutions of rotational and vibrational temperature behind the strong shock waves where $t_{p}$ is the induction time behind a shock wave and $t_{e}$ is defined as the relaxation time of non-equilibrium region, respectively. The $\mathrm{CN}$ emission spectra have been obtained each $200 \mathrm{~ns}$ interval when the shock-heated gas is considered out of equilibrium and each $1 \mu \mathrm{s}$ when equilibrium is assumed. The purple line is the response curve of the No. 3 ion probe, which has a very quick response $(\sim 50 \mathrm{~ns})$ to the arrival of the shock waves and is basically the same for both initial conditions. For the low-pressure condition, the excellent agreement between rotational temperature and vibrational temperature shows that the shock-heated gas should reach equilibrium after $7 \mu \mathrm{s}$ and the equilibrium temperature is equal to $T_{e q}=7000 \pm 400 \mathrm{~K}$, the $t_{p}$ is equal to $0.6 \pm 0.1 \mu \mathrm{s}$, and the $t_{e}$ is equal to $6.2 \pm 0.6 \mu \mathrm{s}$. For the high-pressure condition, the shock-heated gas should reach equilibrium after $5 \mu \mathrm{s}$ and the equilibrium temperature is equal to $T_{e q}=6000 \pm 300 \mathrm{~K}$, and the $t_{p}$ and $t_{e}$ have values of $3.3 \pm 0.5 \mu$ s and $1.5 \pm 0.3$ $\mu \mathrm{s}$, respectively.

\subsection{CO absorption measurements behind the strong shock wave}

Figure 5 presents a typical absorption measurement signal behind a shock wave during one shot in the high pressure condition. In Fig. 5a, a sharp rising voltage output of the No. 3 ion probe indicates the arrival of the shock and propagates to the downstream region of the shock tube. Figure 5b shows a direct-absorption scan using the TDLAS system, in which the laser intensity changes in response to an injection current ramp. To obtain concentration of carbon monoxide, the data processing includes three steps. First, for each measured absorbance signal as shown in Fig. 5b, a polynomial fit to the non-absorbing wings of the absorption feature is used to extrapolate a zero-absorption baseline. Here, the baseline is fitted with a fifth-order polynomial. Second, an absorbance plot of the absorption feature can be constructed based on the baseline obtained in the first step. In our studies, a Voigt profile is used to fit the absorbance plot. The bottom panel of Fig. 6 shows an example of the Voigt fit of the absorption spectra, and the top portion shows the fitting residual, which is less than $1 \%$ of the peak absorption throughout the line profile. Such low fitting-residual illustrates the high SNR achieved in this research. Third, the integrated absorbance can be calculated, and then used to calculate the $\mathrm{CO}$ concentration. The deduced average $\mathrm{CO}$ concentrations in the low and high pressure condition are 2.91 $\times 10^{12} \mathrm{~cm}^{-3}$ and $1.01 \times 10^{13} \mathrm{~cm}^{-3}$, corresponding to equilibrium temperatures equal to $7000 \pm 400 \mathrm{~K}$ and $6000 \pm 300 \mathrm{~K}$, respectively. Moreover, the integrated absorbance of $\mathrm{CO}$ in the non-equilibrium region can also be determined by TDLAS, however, they cannot be used to infer $\mathrm{CO}$ concentration because the $\mathrm{CO}$ number density in the non-equilibrium do not follow the Boltzmann distribution at ground state and intense thermochemical reactions occurred in the non-equilibrium region lead many uncertain factors.

Uncertainty in $\mathrm{CO}$ concentration estimation is also analyzed and evaluated in the following section. Uncertainty in the measurement concentration mainly consists of the uncertainty in temperature measurement and uncertainty in the integrated absorbance. As shown in the Eq. (7), we know temperature is the predominant parameter affecting the calculated $\mathrm{CO}$ concentration and can also affect the accuracy of line-strength calculation. In our experimental 
temperature range, a $4 \%$ uncertainty in the temperature measurement causes a $\sim 5 \%$ the line strength calculation error. The uncertainty in the integrated absorbance is mainly caused by the error associated with boundary layer and Voigt profile fit. The thickness of the boundary is less than $0.5 \mathrm{~mm}$ in our experiments according to the calculation methods from Mirels ${ }^{25}$. The calculated result shows the uncertainty in path length $L$ is $<1 \%$. Consequently, the uncertainty in integrated absorbance estimated in this study is also $<1 \%$. As shown in Fig. 6, the high SNR of the baseline and Voigt profile fit results in uncertainty of $\sim 1 \%$ in the integrated absorbance. Taking into account all of the above uncertainty, the measurement uncertainty in $\mathrm{CO}$ concentration is about $10 \%$.

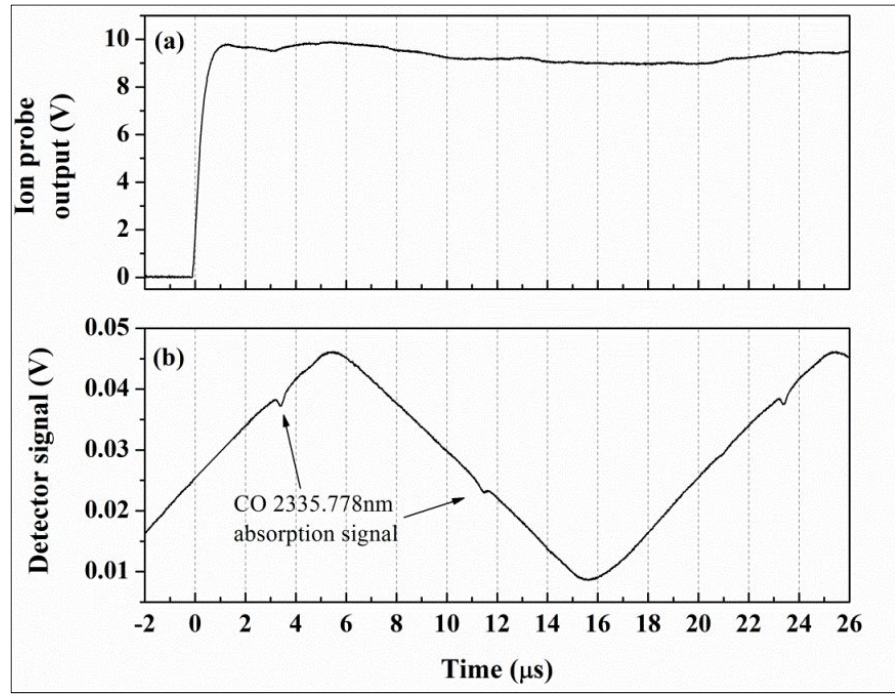

Fig. 5 Raw experimental data behind a strong shock wave under test conditions of $P_{i}=300 \mathrm{~Pa}$ and $V_{s}=5.70 \mathrm{~km} / \mathrm{s}$. (a) No. 3 ion probe output versus time. (b) Detected absorbance signals for a direct absorption scan near $2335.778 \mathrm{~nm}$.

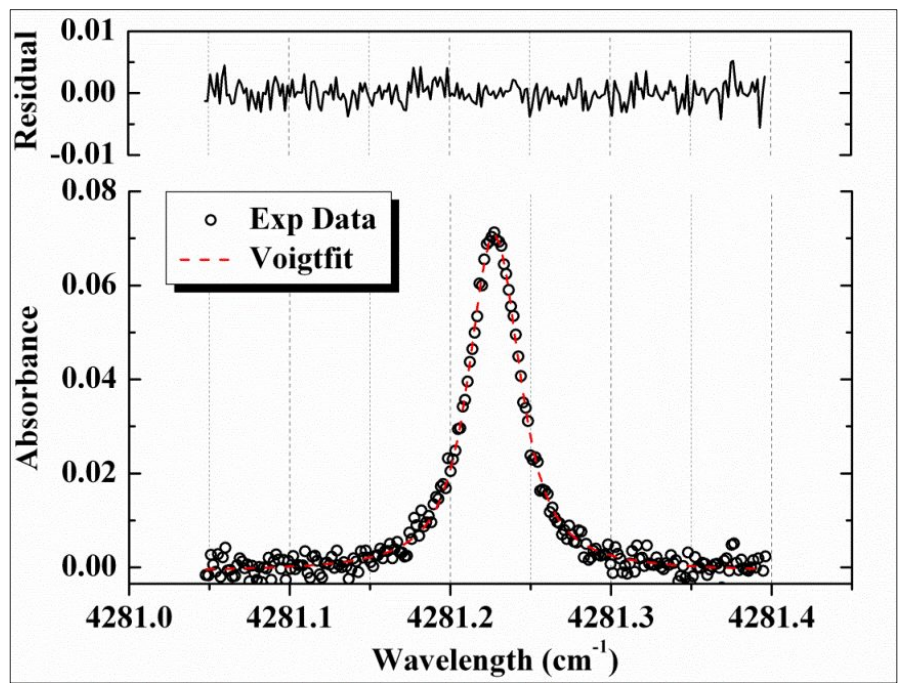

Fig.6 Single-scan absorption data for CO concentration measurement in a hydrogen-oxygen combustion driven shock tube with the CO transition near $2335.778 \mathrm{~nm}$

\section{CONCLUSIONS}

Gas temperature and $\mathrm{CO}$ concentration distribution are diagnosed behind a strong shock wave simulating the Martian atmosphere entry processes by coupling OES and TDLAS. Experiments are carried out in a hydrogen-oxygen combustion driven shock tube with a mixture $\mathrm{CO}_{2}-\mathrm{N}_{2}$ (70:30) and with two sets of initial conditions (100 $\mathrm{Pa}-7.09 \pm$ $0.05 \mathrm{~km} / \mathrm{s}$ and $300 \mathrm{~Pa}-5.68 \pm 0.07 \mathrm{~km} / \mathrm{s}$ ). Time-resolved emission spectroscopy allows an analyzing time evolution of $\mathrm{CN}$ violet system $\left(B^{2} \Sigma^{+} \rightarrow X^{2} \Sigma^{+}, \Delta v=0\right.$ sequence) and rotational and vibrational temperatures from the shock to the 
thermochemical equilibrium. Radiation structure of the shock layer, including induction, relaxation and equilibrium processes are obtained using OES, simultaneously. Meanwhile, tunable diode-laser absorption measurements of CO transitions near $2.33 \mu \mathrm{m}$ are conducted. Scanned-wavelength direct absorption mode is utilized with $50 \mathrm{kHz}$ repetition rate. The TDLAS system is monitored and calibrated through a room temperature reference cavity and a Fabry-Perot interferometer. Combined the temperature results using OES, the average $\mathrm{CO}$ concentration in the thermal equilibrium region is deduced for the concerned test state. Finally, an error analysis has been performed to assess the uncertainties from the temperature measurement and boundary layer.

This work completes our previous work $^{24}$ and illustrates the feasibility the potential of TDLAS system for species concentration measurements during space probe entry phase. Existing experimental results will be very helpful to understand $\mathrm{CO}_{2}$ dissociation and provide verification data of ground-state densities for studying chemical kinetic processes occurring in the shock layer.

\section{REFERENCES}

[1] Brandis, A. M., Johnson, C. O., Cruden, B. A., et al. "Investigation of nonequilibrium radiation for Mars entry," 51st AIAA Aerospace Sciences Meeting including the New Horizons Forum and Aerospace Exposition, AIAA 2013-1055.

[2] Rond, C., Boubert, P. "Nonequilibrium radiation in shocked Martian mixtures," J. Thermophys. Heat Transfer 24(1), 40-49 (2010).

[3] Rond, C., Boubert, P., Félio, J. M., et al. "Radiation measurements in a shock tube for Titan mixtures," J. Thermophys. Heat Transfer 21(3), 638-646 (2007).

[4] Rond, C., Boubert, P., Félio, J.M., et al. "Nonequilibrium radiation behind a strong shock wave in $\mathrm{CO}_{2}-\mathrm{N}_{2}$," Chem. Phys. 340, 93-104 (2007).

[5] Sharma, S. P. and Gillespie, W. "Nonequilibrium and equilibrium shock front radiation measurements," J. Thermophys. Heat Transfer 5(3), 257-265 (1991).

[6] Park, C., Howe, J. T., Jaffe, R. L., et al. "Review of chemical kinetic problems of future NASA mission II: Mars entries," J. Thermophys. Heat Transfer 8(1), 9-23 (1994).

[7] Grinstead, J. H., Wright, M. J., Bogdanoff, D. W., et al. "Shock radiation measurements for Mars aerocapture radiative heating analysis," J. Thermophys. Heat Transfer 23(2), 249-255 (2009).

[8] Dikalyuk, A. S., Surzhikov, S. T., Kozlov, P. V., et al. "Nonequilibrium spectral radiation behind the shock waves in Martian and Earth atmospheres," 44th AIAA Thermophysics Conference, AIAA 2013-2505.

[9] Annaloro, J., Bultel, A., Omaly, P. "Elaboration of a collisional-radiative model for $\mathrm{CO}_{2}-\mathrm{N}_{2}-\mathrm{Ar}$ plasma flows: application to atmosphere Martian entries," 42nd AIAA Thermophysics Conference, AIAA 2011-3954.

[10]Potter, D. F., Eichmann, T., Brandis, A., et al. "Simulation of radiating $\mathrm{CO}_{2}-\mathrm{N}_{2}$ shock layer experiments at hyperbolic entry conditions," 40th Thermophysics Conference, AIAA 2008-3933.

[11]Fujita, K., Sato, S., Abe,T., et al. "Experimental investigation of air radiation from behind a strong shock wave," J. Thermophys. Heat Transfer, 16(1), 77-82 (2002).

[12] Brandis, A. M., Morgan,R. G., McIntyre, T. J., et al. "Nonequilibrium radiation intensity measurements in simulated Titan atmospheres," J. Thermophys. Heat Transfer, 24(2), 291-300 (2010).

[13] Takayanagi, H., Fujita, K. "Absolute radiation measurements behind strong shock wave in carbon dioxide flow for Mars aerocapture missions," 43rd AIAA Thermophysics Conference, AIAA 2012-2744.

[14] Babou, Y., Rivière, P., Perrin, M-Y., et al. "Spectroscopic data for the prediction of radiative transfer in $\mathrm{CO}_{2}-\mathrm{N}_{2}$ plasma," J. Quant. Spectrosc. Ra. 110, 89-108 (2009).

[15] Bose, D., Wright, M. J., Bogdanoff, D. W., et al. "Modeling and experimental assessment of CN radiation behind a strong shock wave," J. Thermophys. Heat Transfer, 20(2), 220-230 (2006).

[16]Lee, E-S., Park, C., Chang, K-S. "Shock Tube determination of CN formation rate in a CO- $\mathrm{N}_{2}$ mixture," J. Thermophys. Heat Transfer, 21(1), 50-56 (2007).

[17] Rond, C., Bultel, A., Boubert, P., et al. "Spectroscopic measurements of nonequilibrium $\mathrm{CO}_{2}$ plasma in RF torch," Chem. Phys. 354, 16-26 (2008).

[18]Lin, X., Yu, X. L., Li F., et al. "Combined TDLAS and OES technique for CO concentration measurement in shock-heated Martian atmosphere," Proc. of SPIE 8796, 87961J-7

[19] Lin, X., Yu, X. L., Li, F., et al. "Measurements of nonequilibrium and equilibrium temperature behind a strong shock wave in simulated Martian atmosphere," Acta. Mech. Sinica. 28(5), 1296-1302 (2012). 
[20]Cipullo, A., Filippis, F. D., Zeni, L. "Temperature measurements of air plasma flow using optical emission spectroscopy," J. Thermophys. Heat Transfer 25(3), 354-360 (2011)

[21] Kurosawa, K., Sugita, S., Fujita, K., et al. "Rotational-temperature measurements of chemically reacting CN using band-tail spectra," J. Thermophys. Heat Transfer 23(3), 463-472 (2009).

[22]Farooq, A., Jeffries, J. B., Hanson, R. K. " $\mathrm{CO}_{2}$ concentration and temperature sensor for combustion gases using diode-laser absorption near $2.7 \mu \mathrm{m}$," Appl. Phys. B 90, 619-628 (2008).

[23] Goorvitch, D. "Infrared CO line list for the $X^{1} \Sigma^{+}$state," Astrophys. J. Suppl. S. 95, 535-552 (1994).

[24]Lin, X., Yu, X. L., Li F., et al. "CO concentration and temperature measurements in a shock tube for martian mixtures by coupling OES and TDLAS," Appl. Phys. B, 110, 401-409 (2012)

[25] Mirels, H. "Turbulent boundary layer behind constant velocity shock including wall blowing effects," AIAA Journal, 22(8), 1042-1047 (1984) 